Research Paper

\title{
Exosomal miR-1305 in the oncogenic activity of hypoxic multiple myeloma cells: a biomarker for predicting prognosis
}

Ji Young Lee ${ }^{1^{*}}$, Daeun Ryu ${ }^{2 *}$, Sung Won Lim ${ }^{3 *}$, Kyung Ju Ryu ${ }^{1}$, Myung Eun Choi ${ }^{1}$, Sang Eun Yoon ${ }^{4}$, Kihyun Kim4, Chaehwa Park ${ }^{1 \bowtie}$ and Seok Jin Kim ${ }^{1,4 \bowtie}$

1. Department of Health Sciences and Technology, Samsung Advanced Institute for Health Sciences and Technology, Sungkyunkwan University, Seoul, Korea.

2. Department of Medical Informatics, College of Medicine, The Catholic University of Korea, Seoul, Republic of Korea.

3. Division of Hematology-Oncology, Department of Medicine, H plus Yangii hospital, Seoul, Korea.

4. Division of Hematology-Oncology, Department of Medicine, Samsung Medical Center, Sungkyunkwan University School of Medicine, Seoul, Korea

*These authors contributed equally as co-first authors.

$\triangle$ Corresponding authors: Seok Jin Kim M.D., Ph.D. Division of Hematology/Oncology, Department of Medicine, Samsung Medical Center, Sungkyunkwan University School of Medicine, 81 Irwon-ro, Gangnam-gu, Seoul 06351, Korea. Tel: +82-2-3410-1766, Fax: +82-2-3410-1754, E-mail: kstwoh@skku.edu; co-corresponding author: Chaehwa Park Ph.D. Department of Health Sciences and Technology, Samsung Advanced Institute for Health Sciences and Technology, Sungkyunkwan University, Seoul, Korea. Tel: +82-2-3410-1766, Fax: +82-2-3410-1754, E-mail: cpark@skku.edu.

(C) The author(s). This is an open access article distributed under the terms of the Creative Commons Attribution License (https://creativecommons.org/licenses/by/4.0/). See http:/ /ivyspring.com/terms for full terms and conditions.

Received: 2020.11.06; Accepted: 2021.03.03; Published: 2021.03.14

\begin{abstract}
Background: Exosomes have emerged as important mediators of tumor progression, and a prognostic role for serum exosomal miRNAs has been suggested in multiple myeloma (MM). Given the association of hypoxia with tumor aggressiveness, including cancer stem cell-like phenotypes, we explored exosomal miRNAs from $M M$ cells under hypoxic conditions and analyzed their diverse roles both in promoting oncogenic activity and in predicting prognosis.

Methods: The human MM cell line, RPMI 8226, was cultured under hypoxic conditions and their exosome production and exosomal miRNA profiles were compared with those of normoxic parental cells. The survival outcome of myeloma patients was compared using serum levels of exosomal miRNAs, and the effects of exosomal miRNAs on the target genes of MM cells and adjacent immune cells were analyzed.

Results: Increased expression of stem cell markers and exosome production were observed in hypoxic MM cells. Exosome miRNA analysis identified a higher expression of miR-1305 in exosomes isolated from hypoxic MM cells than in those of normoxic parental cells. The overall survival of patients with high exosomal miR-1305 was poorer than it was in patients with low exosomal miR-1305. In hypoxic MM cells, an increase of exosomal miR-1305 led to a decrease of cellular miR-1305 and increased expression of the miR-1305 target genes, MDM2, IGFI and FGF2 resulted in the promotion of oncogenic activity of MM. Exosomal miR-1305 was also transferred from $M M$ cells to macrophages, and miR-1305-transferred macrophages showed tumor-promoting, M2-macrophage phenotypes.
\end{abstract}

Conclusions: Exosome-mediated secretion of miR-1305 in MM cells promoted oncogenic activity of hypoxic MM cells and high serum levels of exosomal miR-1305.

Key words: exosome, microRNA, miR-1305, multiple myeloma, hypoxia

\section{Introduction}

Multiple myeloma (MM) is the second most common hematologic malignancy and is characterized by the clonal proliferation of malignant plasma cells in bone marrow (BM) [1, 2]. Despite significant advances in the treatment of $\mathrm{MM}$, including proteasome inhibitors, immunomodulatory drugs and monoclonal antibodies, MM remains incurable because most patients experience recurrent relapse [3-7]. The high frequency of relapse in MM is known to be related to the heterogeneity of tumor cells. Thus, the more aggressive subsets of cells resistant to drugs - the so-called myeloma stem cells could exist in the BM microenvironment resulting in treatment failure in MM patients [8,9]. Although the 
underlying mechanisms of cancer stem cell development are still poorly understood, the acquisition of stem cell-like phenotypes is strongly associated with the adaptation of tumor cells to hypoxic conditions [10, 11]. Indeed, hypoxia in the tumor microenvironment is a well-known factor contributing to tumor progression and drug resistance $[12,13]$. As the oxygen pressure in $\mathrm{BM}$ is lower than that in peripheral blood, the association of hypoxia with a stem cell-like phenotype and with the drug resistance of MM cells has been reported [14, 15].

Exosomes of 30-150 $\mathrm{nm}$ are endosome-derived extracellular vesicles termed 'small extracellular vesicles'; they are cell-derived, small, membranebound particles secreted from various types of cells including tumor cells [16-18]. Tumor cells are able to release exosomes that promote their growth and metastasis, because exosome cargo includes proteins, lipids, DNAs, messenger RNAs and microRNAs (miRNAs) [19, 20]. In particular, small noncoding RNAs are enriched in exosomes, and exosomal miRNA has been suggested as a biomarker that reflects tumor aggressiveness and patient outcomes $[21,22]$. Various miRNA abnormalities have been reported in myeloma patients, suggesting miRNAs play a role in the pathogenesis of MM [23]. For example, expression of the tumor suppressors miR-15a and miR-16 is markedly reduced in MM due to deletion of chromosome 13q14, preventing their ability to inhibit cell proliferation [24]. In contrast, oncogenic miRNAs such as miR-21 and miR-221 are highly expressed, resulting in a decrease of apoptosis and the growth of MM cells $[25,26]$. The expression of exosomal miRNA also shows an association with the expression levels of myeloma-related factors including 32 -microglobulin and IL-6 [27]. Given that exosomal miRNAs can be obtained from peripheral blood, unlike conventional genomic approaches that require a bone marrow biopsy, they could be candidates for use as noninvasive biomarkers in MM. Therefore, we explored exosomal miRNAs from MM cells under hypoxic conditions and analyzed their association with the aggressiveness of MM cells and prognosis in MM patients.

\section{Materials and Methods}

\section{Cell culture}

Human cell lines, RPMI 8226 and THP-1, were purchased from the ATCC (Rockville, MD). Human myeloma RPMI 8226 cells were grown in RPMI 1640 culture medium supplemented with $10 \%$ heatinactivated fetal bovine serum (FBS), penicillin and streptomycin (Gibco-BRL, Grand Island, NY). Human monocytic THP-1 cells were cultured in RPMI 1640 medium containing $10 \%$ heat inactivated FBS and supplemented with $10 \mathrm{mM}$ HEPES, $1 \mathrm{mM}$ pyruvate, $2.5 \mathrm{~g} / \mathrm{L}$ D-glucose, penicillin, streptomycin (Gibco$\mathrm{BRL}$ ) and $50 \mathrm{pM} \beta$-mercaptoethanol (Gibco-BRL).

\section{Hypoxic culture and exosome isolation}

Exosomes were isolated from culture medium using ExoQuick-TC precipitation solution (System Biosciences, Mountain View, CA) according to the manufacturer's instructions as we isolated exosomes from lymphoma cell lines in our previous study [28]. RPMI 8226 cells were cultured for 2 to 4 weeks under hypoxic $\left(2 \% \mathrm{O}_{2}\right.$ and $\left.1 \% \mathrm{O}_{2}\right)$ or normoxic $\left(21 \% \mathrm{O}_{2}\right)$ conditions and then culture medium was replaced with 10\% exosome-depleted FBS (Gibco) for $72 \mathrm{~h}$. Culture medium was collected and differentially centrifuged at $300 \mathrm{~g}$ for $10 \mathrm{~min}, 2000 \mathrm{~g}$ for $10 \mathrm{~min}$ and $10,000 \mathrm{~g}$ at $4{ }^{\circ} \mathrm{C}$ for $30 \mathrm{~min}$ and then passed through a $0.22-\mu \mathrm{m}$ filter to remove cell debris. Clarified cell culture medium was mixed with ExoQuick-TC solution and incubated at $4{ }^{\circ} \mathrm{C}$ overnight before centrifugation twice at 1,500 $\mathrm{g}$ for $30 \mathrm{~min}$ and $5 \mathrm{~min}$, respectively, in order to remove the supernatant. The pellet was resuspended in $100 \mu \mathrm{L}$ of phosphatebuffered saline (PBS).

\section{Exosome isolation from serum samples of myeloma patients}

The study population was selected from myeloma patients enrolled into our previous two cohort studies that were approved by the institutional review board at the Samsung Medical Center (IRB number 2009-08-065 and 2013-09-009). As the cohort study collected serum samples after written informed consent, myeloma patients' serum-derived exosomes could be analyzed for the validation of in vitro cell-line study results. Thus, we selected myeloma patients according to the following inclusion criteria. First, patients should have at least $1 \mathrm{~mL}$ of serum samples that had never been thawed until analysis. Second, patients should have long-term follow-up data for survival analysis. Third, patients should be initially treated with bortezomib- or thalidomide-containing regimens to minimize the heterogeneity of induction treatment. Accordingly, we selected 42 patients who were initially treated with bortezomib- or thalidomide-containing regimens between 2010 and 2017. The last update of survival and disease status was performed in March, 2020. For the isolation of exosomes, serum was differentially centrifuged at $2,000 \mathrm{~g}$ at $4{ }^{\circ} \mathrm{C}$ for $10 \mathrm{~min}$ and $10,000 \mathrm{~g}$ at $4{ }^{\circ} \mathrm{C}$ for 30 $\mathrm{min}$, and passed through a $0.22-\mu \mathrm{m}$ filter to remove cell debris. Clarified serum was mixed with ExoQuick (System Biosciences, Palo Alto, CA) and incubated at $4{ }^{\circ} \mathrm{C}$ for $30 \mathrm{~min}$ then centrifuged twice at $1,500 \mathrm{~g}$ for 30 
min and $5 \mathrm{~min}$, respectively to remove the supernatant. The pellet was resuspended in $200 \mu \mathrm{L}$ of PBS. Our protocol of isolating exosomes from archived patients' serum samples was same as that of our previous study for lymphoma patients [28].

\section{Transmission electron microscopy (TEM) analysis}

Exosomes were fixed in $4 \%$ paraformaldehyde and transferred onto Formvar-carbon-coated TEM grids. Fixed samples were allowed to absorb filter-paper for $20 \mathrm{~min}$ in a dry environment. Grids were rinsed in PBS. After removing the supernatant liquid by filter paper absorption, grids were floated on a drop of $2.5 \% \mathrm{w} / \mathrm{v}$ glutaraldehyde for $5 \mathrm{~min}$. Grids were washed 10 times with distilled water and negative stained with $1 \%$ uranyl acetate for $1 \mathrm{~min}$ and then air dried. Grids were observed with a Hitachi TEM 7700 (Hitachi Inc., Dallas, TX) at $80 \mathrm{kV}$.

\section{Nanoparticle tracking analysis (NTA) of exosomes}

Exosome suspensions with concentrations between $1 \times 10^{7} / \mathrm{mL}$ and $1 \times 10^{9} / \mathrm{mL}$ were examined using a NanoSight NS300 (NanoSight Ltd., Amesbury, UK) particle analyzer and analyzed using NTA software (version 2.3; NanoSight Ltd.) for the size and quantity of particles isolated.

\section{RNA extraction and real-time PCR}

Total RNA was isolated from cells and exosomes using the miRNeasy Micro kit according to the manufacturer's instructions (Qiagen Inc., Valencia, CA). RNA concentration was measured using the NanoDrop ND-100 Spectrophotometer (NanoDrop Technologies, Wilmington, DE). For miRNA expression analysis, $50 \mathrm{ng}$ of RNA was mixed with TaqMan MicroRNA Reverse Transcription Kit reagent containing specific miRNA primers and reverse transcribed according to the manufacturer's instructions (Thermo Scientific, Rockford, IL). Real-time PCR was performed by diluting the complementary cDNA product in $2 \times$ TaqMan Universal Master Mix II and 20× TaqMan microRNA Expression Assay for each mature miRNA to be measured: miR-1305 and miR-21 (Applied Biosystems, Waltham, MA). U6 and cel-miR-39 were used as the normalization controls for cells and extracellular vesicles, respectively, in miRNA expression-level analysis. For quantification of gene expression, $2 \mu \mathrm{g}$ RNA was converted to cDNA using an Omniscript RT kit (Qiagen, Valencia, CA). The cDNA generated was amplified using TaqMan assay for HIF-1 $\alpha$, Nanog, OCT4, SOX2 and $\beta$-actin. Reactions were carried out in a QuantStudio 6 system
(Applied Biosystems) and the results expressed as fold change calculated with the comparative CT $(\Delta \Delta \mathrm{Ct})$ method relative to the control sample. $\beta$-Actin was used as the internal control for mRNA analysis.

\section{RNA sequencing and data analysis}

Whole transcriptome sequencing libraries were constructed using a TruSeq RNA sample preparation v2 kit (Illumina). Sequencing was performed using the 100-bp paired-end mode of the TruSeq Rapid PE Cluster kit and TruSeq Rapid SBS kit (Illumina). The sequencing reads were mapped to the GRCh 37.75 human reference genome by using STAR version 2.4.0 [26]. To quantify gene expression, mapped reads were processed by RSEM version 1.2.18 [29]. The 'edgeR' method was used to identify differentially expressed genes (DEGs) and the results were filtered on the absolute value of the $\log 2$ (fold change) to more than two [30]. We performed gene ontology (GO) analysis using DAVID [31].

\section{Western blot}

Exosomes were lysed in RIPA buffer $(0.5 \%$ sodium deoxycholate, $1 \%$ Nonidet P-40, $150 \mathrm{mM}$ $\mathrm{NaCl}, 50 \mathrm{mM}$ Tris [pH 7.5], 0.1\% sodium dodecyl sulfate [SDS] and $1 \mathrm{mM}$ phenylmethylsulfonyl fluoride [PMSF] ) and cleared by microcentrifugation $\left(13,000 \mathrm{rpm}\right.$ for $10 \mathrm{~min}$ at $\left.4{ }^{\circ} \mathrm{C}\right)$. In total, $50 \mu \mathrm{g}$ protein samples were electrophoresed on a $4 \%-12 \%$ SDS polyacrylamide gel (SDS-PAGE) and transferred to nitrocellulose membranes. The membrane was incubated with blocking solution ( $5 \%$ non-fat milk) for $1 \mathrm{~h}$ and incubated overnight at $4{ }^{\circ} \mathrm{C}$ with primary antibodies including anti-Alix, anti-CD63 and $\beta$-actin (Santa Cruz Biotechnology, CA), and HIF-1 $\alpha$, Akt, p-Akt, p-P65, p-ERK, BCL2 and P21 (Cell Signaling, Danvers, MA). The blot was washed with TBST buffer (50 mM Tris [pH 7.5], $150 \mathrm{mM} \mathrm{NaCl}, 0.05 \%$ Tween 20) and further exposed to horseradish peroxidaseconjugated secondary antibody for $1 \mathrm{~h}$ at room temperature. Proteins were visualized using enhanced chemiluminescence (ECL) reagent (Invitrogen, Carlsbad, CA).

\section{NanoString nCounter analyses}

For each sample analyzed, total exosomal RNA was used for miRNA profiling by a NanoString nCounter ${ }^{\circledR}$ microRNA platform (version 3 ) as per the manufacturer's instructions. nCounter miRNA expression assay count data were normalized. Technical normalization was performed according to guidelines provided by NanoString. The nCounter results were analyzed using nSolver 4.0 software according to the manufacturer's instructions. 


\section{MicroRNA transfection}

RPMI 8226 cells were transfected with miR-1305_ mimic and negative control miRNA (Exiqon, Copenhagen, Denmark) for $48 \mathrm{~h}$ and $72 \mathrm{~h}$ using Lipofectamine $^{\mathrm{TM}}$ RNAiMAX (Invitrogen: Thermo Fisher Scientific, Inc.).

\section{Transwell coculture assay}

THP-1 monocytes $\left(3 \times 10^{5}\right)$ cells were seeded into a 24-well plate (lower chamber) and differentiated with $150 \mathrm{nM}$ phorbol 12-myristate 13-acetate (PMA, Sigma-Aldrich, St. Louis, MO) for $24 \mathrm{~h}$, after which the PMA-containing medium was replaced with $10 \%$ FBS-containing 1× RPMI growth medium for $24 \mathrm{~h}$. Then, RPMI 8226 transfected cells were plated in a Transwell chamber (upper chamber pore size, $0.4 \mu \mathrm{m}$; Corning Costar, Tewksbury, MA). This cell density was chosen in order to have a 1:1 ratio between macrophages and RPMI 8226 cells cocultured in 10\% exosome-depleted FBS culture medium.

\section{Statistics}

The correlations between miRNAs and clinical parameters were analyzed using the chi-square test. The survival outcomes were compared using the log-rank test. The overall survival (OS) was measured from the date of diagnosis to the date of death due to any cause. The OS was censored at the date of the last follow-up visit. Two-sided tests were used in all calculations and $p$ values $<0.05$ were considered significant. Statistical analyses were performed with GraphPad Prism 5.0 (GraphPad Software, Inc., San Diego, CA) and the statistical software package IBM SPSS Statistics version 24.0 (IBM Corp., Armonk, NY).

\section{Results}

\section{Upregulation of exosomal production during hypoxia}

We performed long-term hypoxic culture to mimic the hypoxic microenvironment of bone marrow. Thus, RPMI 8226 cells were cultured under hypoxic conditions $\left(2 \% \mathrm{O}_{2}\right.$ and $\left.1 \% \mathrm{O}_{2}\right)$ for 2 weeks and 4 weeks, and surviving myeloma cells were analyzed for hypoxia-induced phenotypic changes. When we compared the colony formation and expression of cell signaling pathways between myeloma cells cultured at hypoxic $\left(2 \% \mathrm{O}_{2}\right)$ and normoxic conditions $\left(21 \% \mathrm{O}_{2}\right)$, myeloma cells that survived under chronic hypoxia showed increased colony formation (Figure 1A). The expression of hypoxia-inducible factor (HIF)- $1 \alpha$ was upregulated in myeloma cells under hypoxic conditions $\left(2 \% \mathrm{O}_{2}\right.$ and $1 \% \mathrm{O}_{2}$ ), and the upregulation of HIF- $1 \alpha$ was more evident in myeloma cells cultured for 4 weeks compared with those cultured for 2 weeks (Figure 1A). Consistent with the association of hypoxia with stem cell-like characteristics, the expression of Oct4, Sox2 and Nanog genes, which are known as embryonic stem cell markers, was increased in hypoxic conditions compared with normoxic conditions (Figure 1A). In transcriptome analysis, we compared changes between hypoxic and normoxic conditions. Fifty-five genes were differentially expressed between the two groups (Figure 1B). GO analysis confirmed that the genes detected in each group were related to the extracellular exosome (Figure 1C). Comparison of exosome production by hypoxic and normoxic myeloma cells showed there was increased production of exosomes from hypoxic myeloma cells (Figure 1D). Importantly, the size and morphology of exosomes produced by hypoxic myeloma cells were not different from those produced by parental cells at normoxic conditions (Figure 1E).

\section{Exosomal miRNA profiles of hypoxic myeloma cells}

We analyzed the expression of exosomal miRNAs from hypoxic and normoxic myeloma cells. Among the differentially expressed miRNAs, miR-1305, miR-30d-5p and miR-21-5p were in more than twofold greater abundance in the hypoxic exosomes compared with normoxic exosomes (Figure 2A). Based on the upregulated exosomal miRNA profiles of hypoxic myeloma cells, we analyzed the expression of miRNAs using archived serum samples of 42 myeloma patients. The clinical and laboratory characteristics of patients were summarized in Table 1. After diagnosis of myeloma, three induction regimens were used as follows: thalidomidedexamethasone ( $\mathrm{n}=16)$, bortezomib-thalidomidedexamethasone $(n=11)$, and bortezomib-melphalanprednisolone $(n=15)$. At the median follow-up of 85 months (95\% Confidence Interval: 55.1-114.9 months), 19 deaths were confirmed. When we divided patients into high and low groups according to the median read counts of each exosomal miRNA, the group with high exosomal miR-1305 showed a tendency to poorer overall survival when compared with the low exosomal miR-1305 group (Figure 2B). However, exosomal miR-30d-5p and miR-21-5p failed to show any association with overall survival. The comparison of clinical and laboratory characteristics between high and low exosomal miR-1305 groups did not show any significant difference (Table 1). The subgroup analysis of overall survival in 26 patients who were initially treated with bortezomib-containing induction treatment showed a significantly worse overall survival by members of the high exosomal miR-1305 group compared with the low miR-1305 group 
(Figure 2C). We performed in silico analysis to determine possible miR-1305 targets using three database systems including miRDB, miRTaBase and TargetScan. Sixty-four transcripts with conserved miR-1305 binding sites were predicted. Predicted target genes were potentially involved in four major pathways (Ras signaling, FoxO signaling, regulation of stem cells and mTOR signaling) (Figure 2D, Supplementary Figure 1).

Table 1. Characteristics of patients

\begin{tabular}{|c|c|c|c|}
\hline \multirow[t]{2}{*}{ Characteristics } & \multicolumn{2}{|c|}{ ExosomalmiR-1305 } & \multirow[t]{2}{*}{$P$ value } \\
\hline & Low $(n=21)$ & High $(\mathrm{n}=21)$ & \\
\hline \multicolumn{4}{|l|}{ Age } \\
\hline$<60$ & $11(52)$ & $9(43)$ & 0.758 \\
\hline$\geq 60$ & $10(48)$ & $12(57)$ & \\
\hline \multicolumn{4}{|l|}{ Sex } \\
\hline Male & $10(48)$ & $10(48)$ & $>0.999$ \\
\hline Female & $11(52)$ & $11(52)$ & \\
\hline \multicolumn{4}{|l|}{ ISS } \\
\hline Low & $7(33)$ & $10(48)$ & 0.580 \\
\hline Intermediate & $9(43)$ & $8(38)$ & \\
\hline High & $5(24)$ & $3(14)$ & \\
\hline \multicolumn{4}{|l|}{ Serum LD } \\
\hline Normal & $9(43)$ & $15(71)$ & 0.137 \\
\hline Increased & $11(52)$ & $6(29)$ & \\
\hline Unknown & $1(5)$ & $0(0)$ & \\
\hline \multicolumn{4}{|l|}{ Bone marrow plasma cell } \\
\hline$<30 \%$ & $8(38)$ & $8(38)$ & $>0.999$ \\
\hline$\geq 30 \%$ & $13(62)$ & $13(62)$ & \\
\hline \multicolumn{4}{|l|}{ Deletion 13} \\
\hline Absence & $9(43)$ & $16(76)$ & 0.076 \\
\hline Presence & $9(43)$ & $3(14)$ & \\
\hline Unknown & $3(14)$ & $2(10)$ & \\
\hline \multicolumn{4}{|l|}{ Deletion 17p } \\
\hline Absence & $17(81)$ & $19(90)$ & 0.519 \\
\hline Presence & $1(5)$ & & \\
\hline Unknown & $3(14)$ & $2(10)$ & \\
\hline \multicolumn{4}{|l|}{ Translocation $(4 ; 14)$} \\
\hline Absence & $14(67)$ & $18(86)$ & 0.287 \\
\hline Presence & $4(19)$ & $1(5)$ & \\
\hline Unknown & $3(14)$ & $2(10)$ & \\
\hline \multicolumn{4}{|l|}{ Translocation $(\mathbf{1 1} ; 14)$} \\
\hline Absence & $9(43)$ & $11(52)$ & 0.549 \\
\hline Presence & $3(14)$ & $1(5)$ & \\
\hline Unknown & $9(43)$ & $9(43)$ & \\
\hline \multicolumn{4}{|l|}{ Translocation $(14 ; 16)$} \\
\hline Absence & $18(86)$ & $19(90)$ & $>0.999$ \\
\hline Presence & $0(0)$ & $0(0)$ & \\
\hline Unknown & $3(14)$ & $2(10)$ & \\
\hline \multicolumn{4}{|l|}{ Translocation $(14 ; 20)$} \\
\hline Absence & $13(62)$ & $10(48)$ & 0.536 \\
\hline Presence & $0(0)$ & $0(0)$ & \\
\hline Unknown & $8(38)$ & $11(52)$ & \\
\hline \multicolumn{4}{|l|}{ First-line induction treatment } \\
\hline Bortezomib-based treatment & $14(67)$ & $12(57)$ & 0.751 \\
\hline Thalidomide-based treatment & $7(33)$ & $9(43)$ & \\
\hline \multicolumn{4}{|c|}{ Autologous stem cell transplantation } \\
\hline Done & $15(71)$ & $11(52)$ & 0.341 \\
\hline Not done & $6(29)$ & $10(48)$ & \\
\hline \multicolumn{4}{|l|}{ Survival status } \\
\hline Alive & $15(71)$ & $8(38)$ & 0.062 \\
\hline Dead & $6(29)$ & $13(62)$ & \\
\hline
\end{tabular}

High expression of miR-1305 target genes in hypoxic myeloma cells

To validate the role of miR-1305 in myeloma cells during hypoxia, we compared the levels of cellular and exosomal miR-1305 in RPMI 8226 cells after $48 \mathrm{~h}$ of culture under hypoxic conditions (Figure $3 \mathrm{~A}$ ). The level of cellular miR-1305 decreased whereas exosomal miR-1305 increased during hypoxia (Figure 3B). However, the level of cellular miR-1305 was not different from exosomal miR-1305 levels under normoxic conditions (Figure 3C). An increase of hypoxia-related markers, including GLUT1, HIF2- $\alpha$, PDK1 and PHD2 was observed, consistent with long-term hypoxia for 2 and 4 weeks (Figure 3D). Under hypoxic conditions, the cellular expression of miR-1305 targets, including FGF2, IGF1 and MDM2 was upregulated compared with that under normoxic conditions (Figure 3E). This inverse association of cellular miR-1305 with target genes was also observed in miR-1305 transfected RPMI 8226 cells (Supplementary Figure 2). Considering that cellular miR-1305 was downregulated under hypoxia, the upregulation of cellular miR-01305 target gene expression - under hypoxia might be caused by the export of miR-1305 via exosomes (Figure 3F).

\section{Effects of exosomal miR-1305 on macrophages}

To explore the effects of exosomal miR-1305 derived from myeloma cells on immune cells of the tumor microenvironment, we cocultured miR-1305transfected RPMI 8226 cells and THP-1 macrophage cells (Figure 4A). After coculture, we confirmed the movement of RPMI 8226-derived exosomes into THP-1 cells (Figure 4B). Accordingly, intracellular miR-1305 levels increased in RPMI 8226 as well as in THP-1 cells after $48 \mathrm{~h}$ and $72 \mathrm{~h}$ of coculture (Figure 4C). Then we analyzed the expression of M2 macrophage markers in THP- 1 cells after $48 \mathrm{~h}$ and 72 h of coculture with miR-1305-transfected RPMI 8226 cells. Coculture with miR-1305-transfected MM cells induced expression of TGF- $\beta$ and CCL2 in THP- 1 cells (Figure 4D). These results suggest that exosomal miR-1305 may be responsible, at least in part, for the M2 polarization of THP-1 promoting the pro-tumor effects of macrophages (Figure 4E).

\section{Discussion}

Exosomes have been reported to play a role in the tumorigenesis of many cancer types, particularly through transfer of miRNA silencing of messenger RNA in target cells [32]. Although cell-free miRNAs exist in blood, exosomal miRNAs could reflect the biology of tumor cells more exactly because tumor cells secrete exosomes containing miRNAs by active processes, whereas cell-free miRNAs are released from dead tumor cells by apoptosis or necrosis [33]. This study analyzed the effect of hypoxic MM cellderived exosomal miRNAs on tumor aggressiveness 
and its association with poor outcomes in MM patients. Our hypoxia model showed increased expression of stem cell markers in hypoxic MM cells compared with normoxic parental cells, and the overexpression of exosome production-related genes was found in the profiling of differentially expressed genes in hypoxic MM cells. Accordingly, the number of exosomes produced from hypoxic MM cells was significantly higher than that from normoxic parental cells. Our results are consistent with the previous study reporting a twofold increase of exosomes secreted from hypoxia-resistant MM cells cultured at $1 \% \mathrm{O}_{2}$, mimicking in vivo conditions of hypoxic $\mathrm{BM}$ [34].

Our comparison of exosomal miRNAs between hypoxic and normoxic MM cells identified differentially expressed exosomal miRNA profiles of hypoxic MM cells. Thus, the expression of miR-1305 was significantly higher in exosomes isolated from hypoxic MM cells at $1 \%$ and $2 \% \mathrm{O}_{2}$ than those from normoxic parental MM cells (Figure 2A). Although a previous study reported the prognostic relevance of exosomal let-7b and miR-18a for the first time in MM patients [35], exosomal miR-1305 has never been reported in MM cells. Indeed, the tumor-suppressive role of miR-1305 was recently suggested in non-small cell lung cancer (NSCLC) tissues and cell lines [36]. Thus, miR-1305 inhibited the proliferation of NSCLC cells, and MDM2 was proven as a target of miR-1305, consistent with our study. Furthermore, miR-1305 was reported to inhibit the stem cell properties of liver cancer stem cells in human hepatocellular carcinoma [37]. Thus, miR-1305 disrupted the activation of the AKT-signaling pathway and repressed colony formation and tumorigenicity of cancer stem cells. Our study showed the overall survival of patients with high exosomal miR-1305 was poorer than patients with low exosomal miR-1305. This survival difference was more prominent in patients receiving bortezomib-based induction treatment, even though other exosomal miRNAs such as miR-30d-5p and miR-21-5p failed to show a significant association with overall survival (Figure 2B, C). The exploration of miR-1305 targets using the database (miRDB, miRTaBase and TargetScan) for target genes of miRNAs, revealed well-known genes essential for myeloma cell growth and proliferation such as insulin-like growth factor type 1 (IGF-1) and mouse double minute 2 homolog (MDM2) [38, 39]. Furthermore, miR-1305 can target genes regulating pluripotency of stem cells such as fibroblast growth factor 2 (FGF2) which, out of 22 members of the
(A)
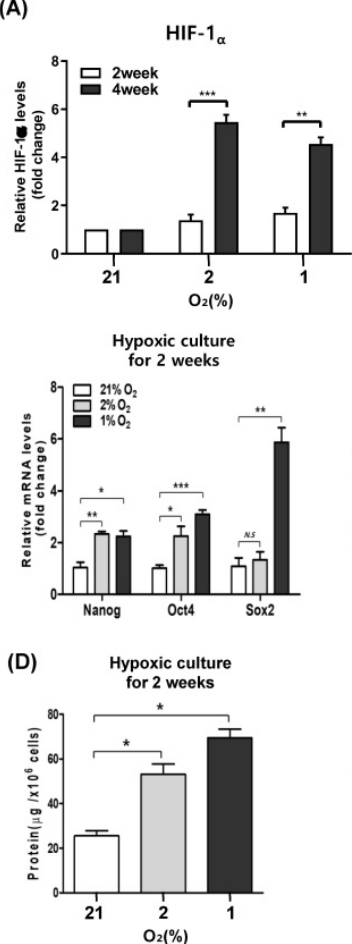

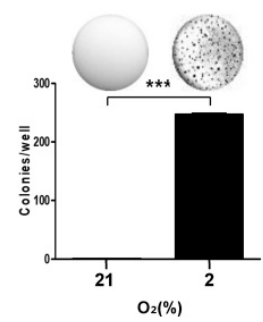

(B)
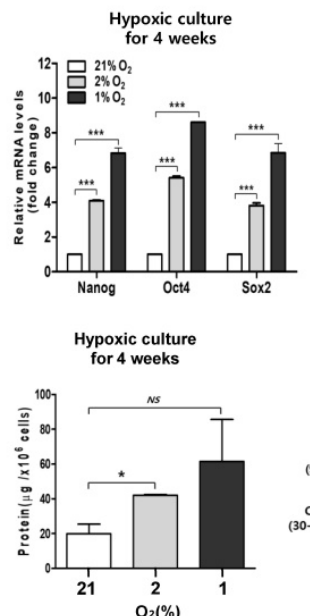
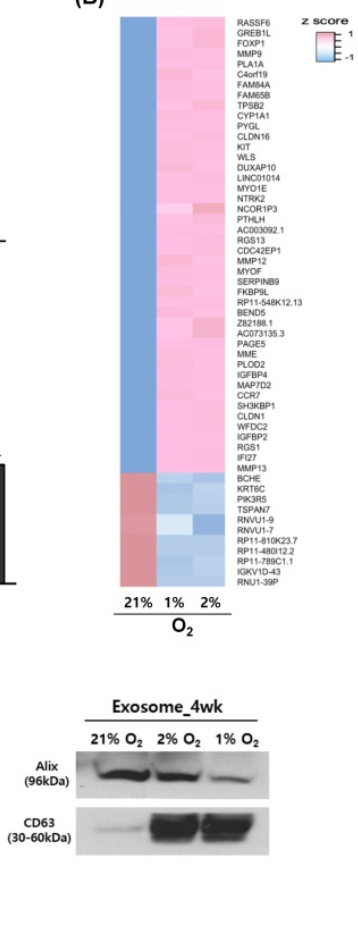

(C)

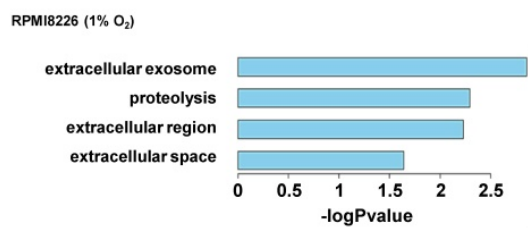

RPMI8226 $\left(2 \% \mathrm{O}_{2}\right)$

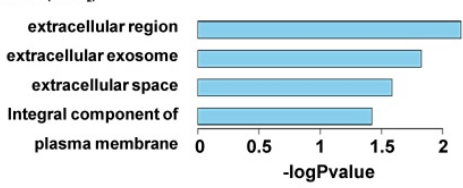

(E)

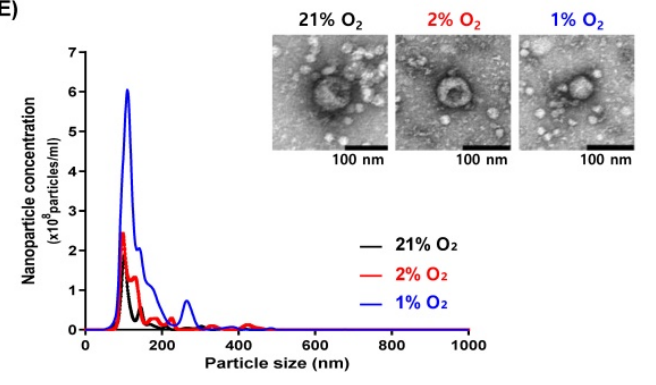

Figure 1. Exosomal production is upregulated during long-term culture of myeloma cells under hypoxia. RPMI 8226 cells were cultured under hypoxic conditions $\left(1 \%\right.$ or $\left.2 \% \mathrm{O}_{2}\right)$ or normoxic conditions $\left(21 \% \mathrm{O}_{2}\right)$ for the indicated times. (A) mRNA expression of HIF-1 $\alpha$ and stem cell markers (Nanog, Oct4 and SOX2) were analyzed by RT-qPCR. Increased colony formation was found in hypoxic RPMI 8226 cells. The data represent the mean \pm SEM of three independent experiments, ${ }^{*} p<0.05$; ** $p<0.01 ; * * * p<$ 0.001 . NS, not significant. (B) Heat map of the differentially expressed genes (DEGs) in both hypoxia conditions. (C) Gene ontology (GO) enrichment analysis for DEGs using DAVID. The bar graph shows GO term with the overlap ratio of more than $10 \%$ between DEGs and GO genes. (D) Protein concentration of exosomes derived from RPMI 8226 cells cultured under hypoxic or normoxic conditions for the indicated times. Western blot analysis of the exosomal markers Alix and CD63 from RPMI 8226 cells cultured for 4 weeks. (E) Representative particle concentration and size distribution of exosomes derived from RPMI 8226 cells cultured under hypoxic or normoxic conditions. Transmission electron microscopic images of exosomes (Scale bar: $100 \mathrm{~nm}$ ). 
human FGF family, was uniquely expressed in the majority of MM cell lines [40]. Instead, miR-1305 was reported to have a regulatory role in the pluripotency of human embryonic stem cells because overexpression of miR-1305 induced stem cell differentiation [41].
(A)

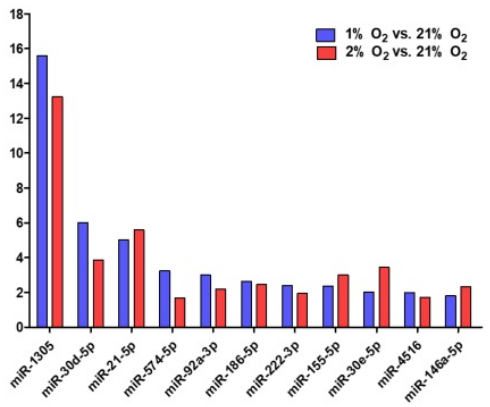

(C)

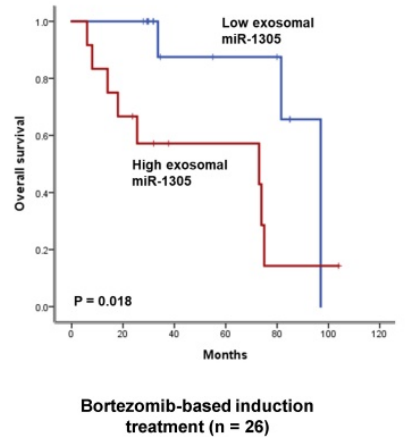

treatment $(n=26)$
(B)

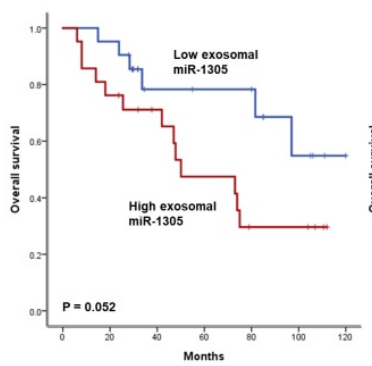

(D)

$(\mathrm{D})$

D)

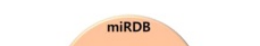

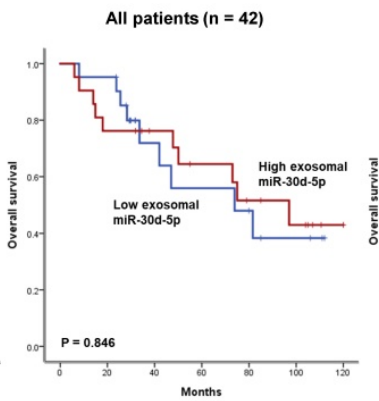
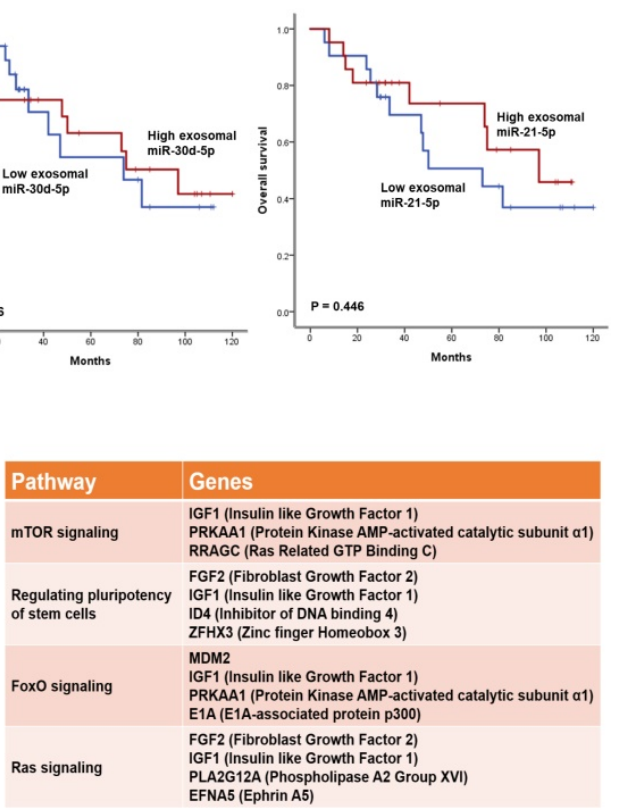

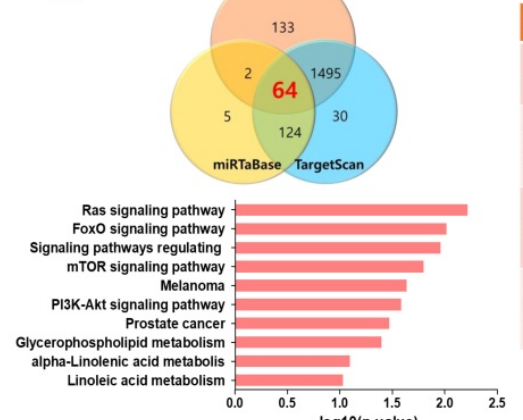

Figure 2. miRNA profiles of hypoxic exosome and its association with overall survival rates in MM patients. (A) Exosomal miRNA profiles of RPMI 8226 cells cultured under hypoxic conditions ( $1 \%$ or $\left.2 \% \mathrm{O}_{2}\right)$. Fold change vs normoxic conditions $\left(21 \% \mathrm{O}_{2}\right)$. (B) Kaplan-Meier curves representing the correlation between miRNA signature based on exosomal miR-1305, miR-30d-5p and miR-21-5p expression levels and overall survival rates in MM patients. (C) MM patients who were initially treated with bortezomib-containing chemotherapy showed a significantly poorer overall survival rate in the high exosomal miR-1305 group. (D) Target gene prediction of differentially expressed miR-1305. Venn diagram showing the overlap of miR-1305 target genes with a high rate of context score by using three different miRNA target analysis programs. Representative pathways of miR-1305 target genes are summarized.

(A)

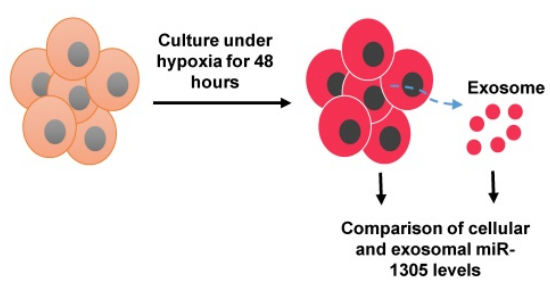

(D)

Expression of hypoxia markers in RPMI8226 after 48 hours of hypoxia

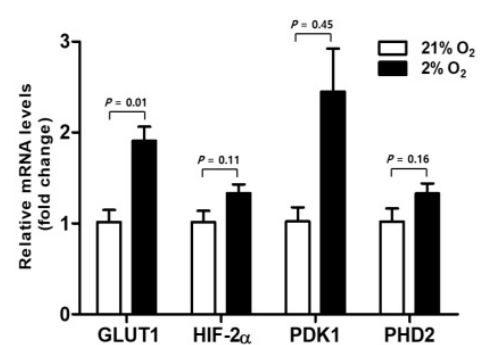

(B)
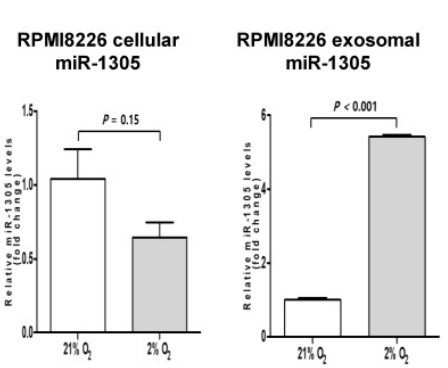

(E)

Cellular expression of miR-1305 target genes in RPMI8226 after 48 hours of hypoxia

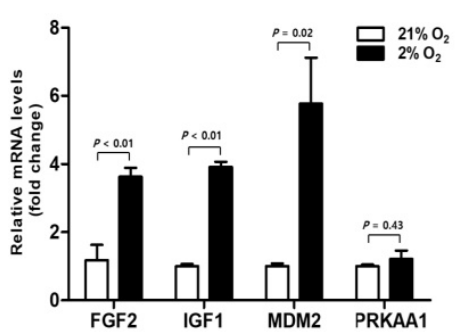

(C)
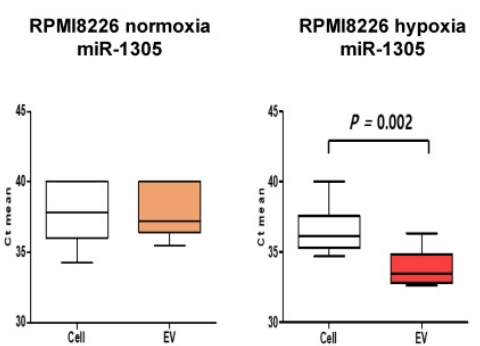

$(\mathrm{F})$

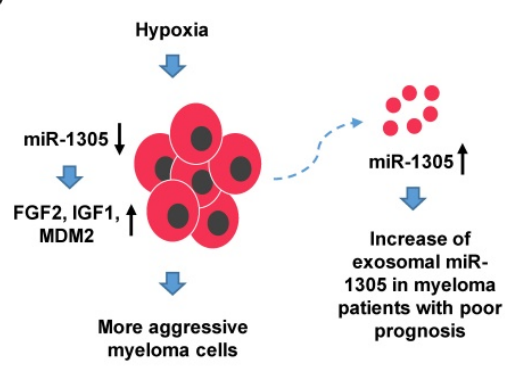

Figure 3. Hypoxia-induced downregulation of cellular miR-1305 and upregulation of miR-1305 target genes in MM cells. RPMI 8226 cells were cultured under normoxic conditions $\left(21 \% \mathrm{O}_{2}\right)$ or hypoxic conditions $\left(2 \% \mathrm{O}_{2}\right)$ for $48 \mathrm{~h}$. (A) Schematic model for comparison of cellular and exosomal miR-1305 from RPMI 8226 cells cultured under hypoxia. (B) The level of cellular and exosomal miR-1305 was analyzed by RT-qPCR. Cellular miR-1305 level was decreased, but exosomal miR-1305 level was increased under hypoxia. (C) Cellular expression of hypoxia markers (GLUT1, HIF2 $\alpha$, PDK1 and PHD2) was upregulated in RPMI 8226 cells cultured under hypoxia. (D) Cellular expression of the miR-1305 candidate targets (FGF2, IGF1 and MDM2) in RPMI 8226 cells cultured under hypoxia. (E) Schematic model for hypothetical consequences caused by myeloma cells cultured under hypoxia. Upregulation of miR-1305 target gene expression was caused by reduced cellular miR-1305 in hypoxic RPMI 8226 cells, which may lead to more aggressive myeloma cells and myeloma patients with poor prognosis. 
(A)

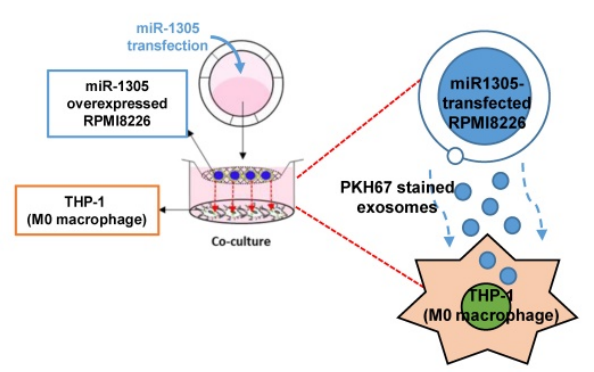

(C)

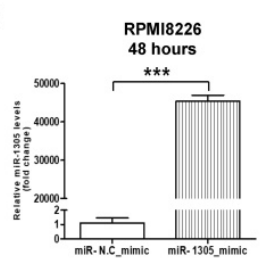

THP-1

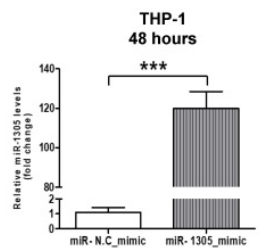

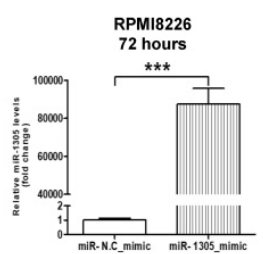

THP-1

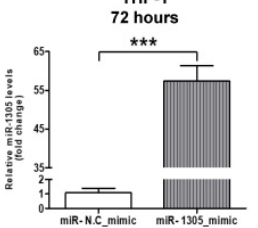

(B)

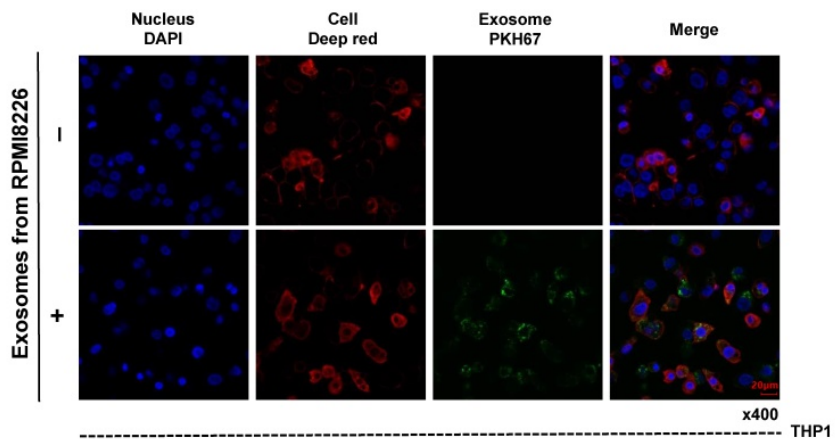

(D)

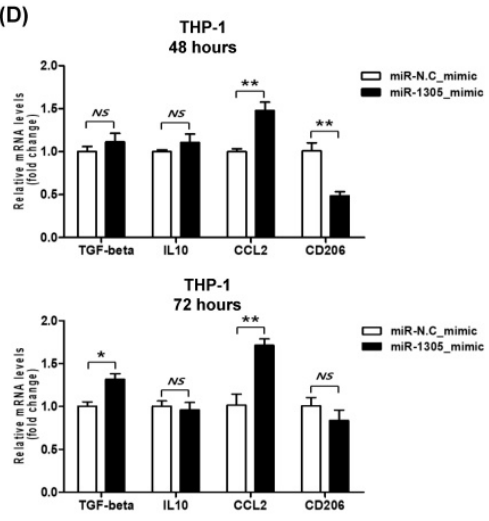

(E)

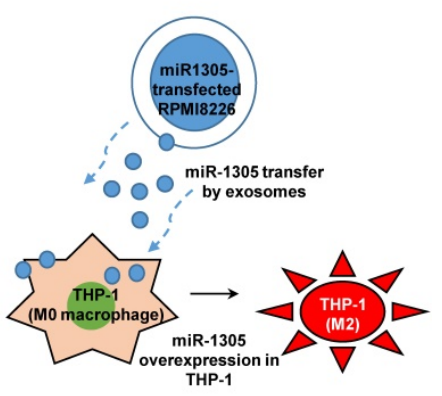

Figure 4. Transfer of miR-135b derived from myeloma cells to THP-1 macrophages via exosomes. (A) Schematic model of the experiment to confirm the effects of exosomal miR-1305 on the tumor microenvironment. THP-1 macrophage cells were seeded in the lower chamber of a Transwell plate, while miR-1305-transfected RPMI 8226 myeloma cells were seeded on the top of the insert. (B) Internalization of myeloma cell-derived exosomes by THP-1 cells. THP-1 macrophage cells were cultured with PKH67-labeled exosomes derived from RPMI 8226 cells. Representative confocal microscopy image shows the PKH67-labeled exosome (green) signals detected in the cytoplasm of THP-1 macrophage cells (red). Nuclear counterstaining was performed using DAPI (blue). (C) Quantitative RT-PCR for miR-1305 was carried out using RNA isolated from RPMI 8226 and THP-1 cells after $48 \mathrm{~h}$ and $72 \mathrm{~h}$ of coculture. The data represent the mean \pm SEM of three independent experiments. $* * * p<0.001$. (D) Expression of M2 macrophage markers in THP-1 cells in response to exosomes derived from RPMI 8226/miR-1305_mimic or control (RPMI 8226/miR-NC_mimic). Quantitative RT-PCR for TGF- $\beta$, IL 10, CCL2 and CD206 was carried out using RNA isolated from THP-1 cells after $48 \mathrm{~h}$ or $72 \mathrm{~h}$ of coculture. TGF- $\beta$ and CCL2 expression was upregulated by the addition of RPMI 8226/miR-1305_mimic exosomes compared with control. (E) Proposed model in this study for how myeloma-derived miR-1305 may promote exosome-induced macrophage polarization in multiple myeloma.

Our study found an increase of miR-1305 in exosomes and a decrease of cellular miR-1305 under hypoxia even though there was no difference between the cellular and exosomal levels of miR-1305 under normoxic conditions (Figure 3B, C). Accordingly, increased expression of cellular IGF1, MDM2 and FGF2, all targets of miR-1305, was observed under hypoxic compared with normoxic conditions (Figure $3 \mathrm{E})$. These findings imply that hypoxia decreases cellular levels of miR-1305 via the exosome-mediated export of miR-1305, leading to increased expression of cellular IGF1, MDM2 and FGF2. Consistent with a pro-tumor effect of exosome-mediated export of tumor-suppressive miR-1305, the aforementioned study also showed that patients with high exosomal let-7 had better survival rates than patients with low exosomal let-7 [35]. Because let-7 acting as a tumor suppressor could inhibit oncogenes such as CCND1, MYC and RAS, decrease of cellular let-7 may lead to cell proliferation [35, 42]. Indeed, exosome-mediated secretion of tumor-suppressor miRNA has been reported as a mechanism to coordinate activation of metastasis as well as cellular disposal of tumorsuppressor miRNAs in other cancer types [43-45].
Thus, hypoxic MM cells could maintain their growth and cancer stem cell-like characteristics via exosomemediated disposal of cellular miR-1305 under hypoxia (Figure 3F). Cell-to-cell communication between MM cells and BM stromal cells is another important function of exosomal miRNAs because they enhance tumor aggressiveness, including angiogenesis [46]. A previous study demonstrated that exosomal miR-135b released from hypoxia-resistant MM cells suppressed target endothelial cells, leading to enhanced endothelial tube formation under hypoxia [34]. The tumor microenvironment comprises fibroblasts, stromal cells and immune cells such as macrophages. In our study, miR-1305 was transferred from MM cells to macrophages and miR-1305-transferred macrophages showed tumor-promoting M2 macrophage phenotypes (Figure 4B, C, D). These results suggest that another function of exosomal miR-1305 is to influence adjacent immune cells of the tumor microenvironment (Figure 4E).

In conclusion, we demonstrated that exosomemediated secretion of miR-1305 in MM cells under hypoxia promoted tumor aggressiveness. We also found unfavorable survival outcomes in myeloma 
patients with high circulating exosomal miR-1305. Considering that exosomal miRNAs may represent the biology of myeloma more accurately than cell-free miRNAs, the assessment of exosomal miR-1305 may be useful for predicting disease outcome and for establishing a risk-adapted treatment approach for myeloma patients. Further studies are required to fully elucidate the underlying mechanisms of the function of exosomal miR-1305 and its use as a biomarker in the clinic.

\section{Supplementary Material}

Supplementary figures and tables. http://www.jcancer.org/v12p2825s1.pdf

\section{Acknowledgements}

This study was supported by the Basic Science Research Program through the National Research Foundation of Korea (NRF) funded by the Ministry of Education, Science, and Technology (NRF-2020R1F1A1073826).

\section{Author contributions}

JYL, DR and SWL wrote the manuscript. JYL, DR, KJR, MEC and SJK analyzed the data. KJR, JYL, DR and MEC performed experiments. SEY and KK reviewed the clinical data. $\mathrm{CP}$ and SJK designed the study.

\section{Competing Interests}

The authors have declared that no competing interest exists.

\section{References}

1. Kyle RA, Gertz MA, Witzig TE, et al. Review of 1027 patients with newly diagnosed multiple myeloma. Mayo Clin Proc. 2003; 78: 21-33.

2. Palumbo A, Anderson K. Multiple myeloma. N Engl J Med. 2011; 364: 1046-1060.

3. Siegel DS, Dimopoulos MA, Ludwig H, et al. Improvement in Overall Survival With Carfilzomib, Lenalidomide, and Dexamethasone in Patients With Relapsed or Refractory Multiple Myeloma. J Clin Oncol. 2018; 36: 728-734.

4. Dimopoulos MA, Oriol A, Nahi H, et al. Daratumumab, Lenalidomide, and Dexamethasone for Multiple Myeloma. N Engl J Med. 2016; 375: 1319-1331.

5. Lonial S, Dimopoulos M, Palumbo A, et al. Elotuzumab Therapy for Relapsed or Refractory Multiple Myeloma. N Engl J Med. 2015; 373: 621-631.

6. Moreau P, Masszi T, Grzasko N, et al. Oral Ixazomib, Lenalidomide, and Dexamethasone for Multiple Myeloma. N Engl J Med. 2016; 374: 1621-1634.

7. Fonseca $\mathrm{R}$, Abouzaid $\mathrm{S}$, Bonafede $\mathrm{M}$, et al. Trends in overall survival and costs of multiple myeloma, 2000-2014. Leukemia. 2017; 31: 1915-1921.

8. Hajek R, Okubote SA, Svachova H. Myeloma stem cell concepts, heterogeneity and plasticity of multiple myeloma. Br J Haematol. 2013; 163: 551-564.

9. Gao M, Kong Y, Yang G, et al. Multiple myeloma cancer stem cells. Oncotarget. 2016; 7: 35466-35477.

10. Axelson H, Fredlund E, Ovenberger $M$, et al. Hypoxia-induced dedifferentiation of tumor cells--a mechanism behind heterogeneity and aggressiveness of solid tumors. Semin Cell Dev Biol. 2005; 16: 554-563.

11. Keith B, Simon MC. Hypoxia-inducible factors, stem cells, and cancer. Cell. 2007; 129: 465-472.
12. Vaupel P, Mayer A, Hockel M. Tumor hypoxia and malignant progression. Methods Enzymol. 2004; 381: 335-354.

13. Quail DF, Joyce JA. Microenvironmental regulation of tumor progression and metastasis. Nat Med. 2013; 19: 1423-1437.

14. Muz B, de la Puente P, Azab F, et al. Hypoxia promotes stem cell-like phenotype in multiple myeloma cells. Blood Cancer J. 2014; 4: e262.

15. Azab AK, Hu J, Quang $\mathrm{P}$, et al. Hypoxia promotes dissemination of multiple myeloma through acquisition of epithelial to mesenchymal transition-like features. Blood. 2012; 119: 5782-5794.

16. Raposo G, Stoorvogel W. Extracellular vesicles: exosomes, microvesicles, and friends. J Cell Biol. 2013; 200: 373-383.

17. Thery C, Witwer KW, Aikawa E, et al. Minimal information for studies of extracellular vesicles 2018 (MISEV2018): a position statement of the International Society for Extracellular Vesicles and update of the MISEV2014 guidelines. J Extracell Vesicles. 2018; 7: 1535750.

18. Sharma A, Khatun Z, Shiras A. Tumor exosomes: cellular postmen of cancer diagnosis and personalized therapy. Nanomedicine (Lond). 2016; 11: 421-437.

19. Yeh YY, Ozer HG, Lehman AM, et al. Characterization of CLL exosomes reveals a distinct microRNA signature and enhanced secretion by activation of BCR signaling. Blood. 2015; 125: 3297-3305.

20. Azmi AS, Bao B, Sarkar FH. Exosomes in cancer development, metastasis, and drug resistance: a comprehensive review. Cancer Metastasis Rev. 2013; 32: 623-642.

21. Kinoshita T, Yip KW, Spence T, et al. MicroRNAs in extracellular vesicles: potential cancer biomarkers. J Hum Genet. 2017; 62: 67-74.

22. An $\mathrm{T}, \mathrm{Qin} \mathrm{S}, \mathrm{Xu} \mathrm{Y}$, et al. Exosomes serve as tumour markers for personalized diagnostics owing to their important role in cancer metastasis. J Extracell Vesicles. 2015; 4: 27522.

23. Handa H, Murakami Y, Ishihara R, et al. The Role and Function of microRNA in the Pathogenesis of Multiple Myeloma. Cancers (Basel). $2019 ; 11$.

24. Lerner M, Harada M, Loven J, et al. DLEU2, frequently deleted in malignancy, functions as a critical host gene of the cell cycle inhibitory microRNAs miR-15a and miR-16-1. Exp Cell Res. 2009; 315: 2941-2952.

25. Leone E, Morelli E, Di Martino MT, et al. Targeting miR-21 inhibits in vitro and in vivo multiple myeloma cell growth. Clin Cancer Res. 2013; 19 : 2096-2106.

26. Di Martino MT, Gulla A, Cantafio ME, et al. In vitro and in vivo anti-tumor activity of miR-221/222 inhibitors in multiple myeloma. Oncotarget. 2013; 4: 242-255.

27. Zhang ZY, Li YC, Geng CY, et al. Potential Relationship between Clinical Significance and Serum Exosomal miRNAs in Patients with Multiple Myeloma. Biomed Res Int. 2019; 2019: 1575468.

28. Ryu KJ, Lee JY, Choi ME, et al. Serum-Derived Exosomal MicroRNA Profiles Can Predict Poor Survival Outcomes in Patients with Extranodal Natural Killer/T-Cell Lymphoma. Cancers (Basel). 2020; 12

29. Li B, Dewey CN. RSEM: accurate transcript quantification from RNA-Seq data with or without a reference genome. BMC Bioinformatics. 2011; 12: 323

30. Robinson MD, McCarthy DJ, Smyth GK. edgeR: a Bioconductor package for differential expression analysis of digital gene expression data. Bioinformatics. 2010; 26: 139-140.

31. Huang da W, Sherman BT, Lempicki RA. Systematic and integrative analysis of large gene lists using DAVID bioinformatics resources. Nat Protoc. 2009; 4: 44-57.

32. Melo SA, Sugimoto $\mathrm{H}, \mathrm{O}^{\prime}$ Connell JT, et al. Cancer exosomes perform cell-independent microRNA biogenesis and promote tumorigenesis. Cancer Cell. 2014; 26: 707-721.

33. Cortez MA, Bueso-Ramos C, Ferdin J, et al. MicroRNAs in body fluids--the mix of hormones and biomarkers. Nat Rev Clin Oncol. 2011; 8: 467-477.

34. Umezu T, Tadokoro H, Azuma K, et al. Exosomal miR-135b shed from hypoxic multiple myeloma cells enhances angiogenesis by targeting factor-inhibiting HIF-1. Blood. 2014; 124: 3748-3757.

35. Manier S, Liu CJ, Avet-Loiseau H, et al. Prognostic role of circulating exosomal miRNAs in multiple myeloma. Blood. 2017; 129: 2429-2436.

36. Cai $Y$, Hao $Y$, Ren $H$, et al. miR-1305 Inhibits The Progression Of Non-Small Cell Lung Cancer By Regulating MDM2. Cancer Manag Res. 2019; 11: 9529-9540.

37. Wei X, You X, Zhang J, et al. MicroRNA-1305 Inhibits the Stemness of LCSCs and Tumorigenesis by Repressing the UBE2T-Dependent Akt-Signaling Pathway. Mol Ther Nucleic Acids. 2019; 16: 721-732.

38. Sprynski AC, Hose D, Caillot L, et al. The role of IGF-1 as a major growth factor for myeloma cell lines and the prognostic relevance of the expression of its receptor. Blood. 2009; 113: 4614-4626.

39. Elnenaei $\mathrm{MO}$, Gruszka-Westwood AM, A'Hernt $\mathrm{R}$, et al. Gene abnormalities in multiple myeloma; the relevance of TP53, MDM2, and CDKN2A. Haematologica. 2003; 88: 529-537. 
40. Krejci P, Mekikian PB, Wilcox WR. The fibroblast growth factors in multiple myeloma. Leukemia. 2006; 20: 1165-1168.

41. Jin S, Collin J, Zhu L, et al. A Novel Role for miR-1305 in Regulation of Pluripotency-Differentiation Balance, Cell Cycle, and Apoptosis in Human Pluripotent Stem Cells. Stem Cells. 2016; 34: 2306-2317.

42. Manier S, Powers JT, Sacco A, et al. The LIN28B/let-7 axis is a novel therapeutic pathway in multiple myeloma. Leukemia. 2017; 31: 853-860.

43. Kanlikilicer P, Rashed MH, Bayraktar R, et al. Ubiquitous Release of Exosomal Tumor Suppressor miR-6126 from Ovarian Cancer Cells. Cancer Res. 2016; 76: 7194-7207.

44. Rashed MH, Kanlikilicer P, Rodriguez-Aguayo C, et al. Exosomal miR-940 maintains SRC-mediated oncogenic activity in cancer cells: a possible role for exosomal disposal of tumor suppressor miRNAs. Oncotarget. 2017; 8: 20145-20164.

45. Ostenfeld MS, Jeppesen DK, Laurberg JR, et al. Cellular disposal of miR23b by RAB27-dependent exosome release is linked to acquisition of metastatic properties. Cancer Res. 2014; 74: 5758-5771.

46. Wan Z, Gao X, Dong Y, et al. Exosome-mediated cell-cell communication in tumor progression. Am J Cancer Res. 2018; 8: 1661-1673. 